LA MERCADOTECNIA EN LA RED SOCIAL "FACEBOOK".

\title{
La Mercadotecnia en la Red Social "Facebook"
}

\author{
Marketing in the Facebook Social Media
}

Fuentes Frías, Fred Alexander *, García Rodríguez, José Félix **

*Estudiante de Licenciatura en Mercadotecnia. División Académica de Ciencias Económico Administrativas de la Universidad Juárez Autónoma de Tabasco. ORCID: https://orcid.org/0000-00030411-3101.

**Doctor en Finanzas Públicas por la Facultad de Economía de la Universidad Veracruzana. ProfesorInvestigador de la Universidad Juárez Autónoma de Tabasco. Email: jfgr@hotmail.com, ORCID: https://orcid.org/0000-0002-7319-1472.

Correo para recibir correspondencia: alexander.fuentes.frias@gmail.com 
LA MERCADOTECNIA EN LA RED SOCIAL "FACEBOOK".

\section{RESUMEN}

La constante evolución de la tecnología y la mayor facilidad con que las personas pueden acceder al internet, han creado el escenario perfecto para la mercadotecnia digital, en esta investigación se expone la situación que se presenta en plataforma Facebook, conocida a nivel mundial y actualmente, una de las redes sociales más influyentes e importantes, ésta misma ha tomado una mayor relevancia en el ámbito de compra, venta y contacto entre vendedor y cliente por lo que su uso como herramienta de marketing es cada vez más indispensable.

OBJETIVO: Conocer el nivel de efectividad del marketing en la plataforma de red social Facebook, para llevar a cabo el proceso de compra y promoción de los productos.

MATERIAL Y MÉTODO: El enfoque fue cualitativo, utilizando las técnicas de recolección en campo mediante una encuesta flash digital a usuarios de esta red social. Además, se desarrolló trabajo de investigación documental.

RESULTADOS: Los usuarios de esta red social son muy perceptibles en forma positiva las diversas publicidades lanzadas en esta plataforma, fundamentándose en la base de datos que cuenta esta red social, que se le hace llegar de acuerdo con sus gustos.

CONCLUSIONES: Los hallazgos de la investigación muestran una alta percepción entre los usuarios ante la publicidad en esta red social, confirmando la investigación documental con los resultados por el sondeo, dando como resultado un gran incremento del marketing en internet.

PALABRAS CLAVE: Red Social. Facebook. Mercadotecnia. Publicidad. Internet.

\section{ABSTRACT}

The constant evolution of technology and the greater ease in which people can have access to the internet, have created the perfect setting for digital marketing. In this research, the Facebook platform is presented as one of the most influential and important social media that has become the most relevant in the field of purchase, sale and contact between sellers and customers, as it has become an ever-growing marketing tool.

OBJECTIVE: To know the level of marketing effectiveness in the Facebook social media, in order to carry out the process of purchasing and promoting products. 
LA MERCADOTECNIA EN LA RED SOCIAL "FACEBOOK".

MATERIAL AND METHOD: Study carried out with a qualitative approach, by doing a documentary review and using the field information collection through a flash survey to Facebook users.

RESULTS: Facebook users are perceptible in a positive way to several advertisements launched in the platform, and selected based on their preferences.

CONCLUSIONS: The research findings show a high perception among users to advertisements in this social media, thus confirming the information collected through the documentary review with the results of the flash survey, resulting in a large increase in internet marketing.

KEY WORDS: Social media. Facebook. Marketing. Advertisement. Internet.

\section{INTRODUCCIÓN}

El impacto en la red social más famosa de la población ha sido de manera positiva, puesto que, las empresas invierten de manera considerable en ella para la publicidad de sus productos y/o servicios, lo que provoca un incremento en sus ventas y mayor alcance a clientes y clientes potenciales.

Existe la discusión sobre sí las redes sociales son buenas o malas y cuál debería ser el uso correcto que se le dé. En este sentido, se puede considerar que las redes sociales, a pesar de representar un cambio muy grande en ciertos ámbitos, son muy buenas siempre y cuando las usemos de una forma que sea benéfica para uso personal y para las empresas que la utilizan, se pueden usar como un punto de encuentro para generar discusión y crear opinión sobre la actualidad, no solo se pueden usar como un medio de difusión masiva o una forma de comunicarnos con cualquier persona, las redes han roto casi por completo las barreras tempo/espaciales, por lo tanto, se puede difundir publicidad con alguien que esté al otro lado del mundo sin problema alguno.

Como referencia de esta información, que indica el índice de inversión en marketing en redes sociales se va incrementado en los siguientes años, tal y como se observa en la siguiente Figura. 


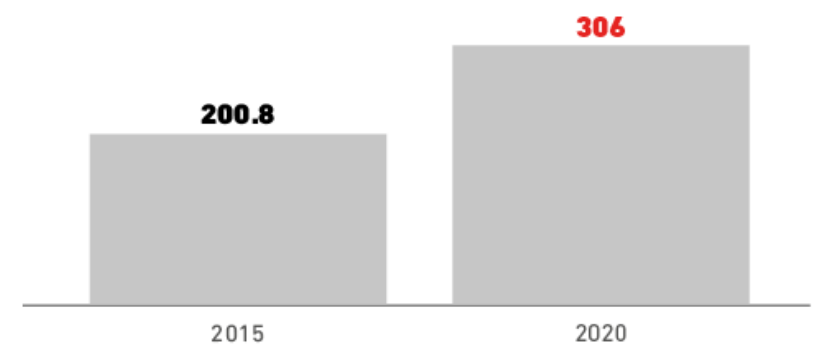

Figura 1. Inversiones en marketing digital en el mundo, comparativo 2015-2020.

Fuente: (Garibay, 2017).

De acuerdo con datos de Facebook, los perfiles de empresas en esta plataforma se han incrementado un 33 por ciento durante los últimos años, para llegar a un total aproximado de 60 millones de páginas de negocio en la red social (González, 2018).

Facebook logra crecer 70 millones de usuarios activos en un solo trimestre, cifra que no se había alcanzado en toda la historia de esta red social, las imágenes son responsables del 75 al 90 por ciento de la eficacia de los anuncios en Facebook el desempeño de los anuncios en esta depende de una variedad de factores, incluida tu industria, presupuesto total, tipo de campaña, público objetivo y cuánto tiempo esté activa tu publicidad ya que muchos de los usuarios de Facebook ven videos sin el sonido, los videos subtitulados pueden incrementar las vistas en tiempo real en un promedio del 12 por ciento (Newberry, 2020).

\section{MARCO TEORICO}

\section{¿Qué es Facebook?}

De acuerdo con Gonçalves (2020), los inicios de Facebook se dieron en el 2003 en la Universidad de Harvard bajo el nombre de Facemash creado por Mark Zuckerberg, Chris Hughes, Dustin Moskovitz y Eduardo Saverin (de origen brasileño), todos estudiaban el segundo año de la universidad. El principal objetivo en aquel entonces fue identificar cuáles eran las chicas más atractivas del campus y en consecuencia las involucradas se sintieron ofendidas puesto que la información la habían obtenido del sistema de seguridad de la universidad y, esto, a su vez alarmó a los ejecutivos de Harvard quienes de inmediato cerraron el sitio web. Más tarde, Mark inició a programar un nuevo código bajo el nombre de "The Facebook". A través de este portal se podía crear un vínculo de amistad en modalidad virtual entre universitarios del campus y universidades cercanas. 
LA MERCADOTECNIA EN LA RED SOCIAL "FACEBOOK".

Para el año 2005, Mark Zuckerberg, Sean Parker (cofundador de Nepster) se inauguró oficialmente a la red social con el nombre que actualmente se conoce "Facebook" y fue así que, en el 2006 se liberó el acceso a jóvenes estudiantes de secundaria y para trabajadores de empresas a nivel general y poco después cualquier persona que tuviera más de 13 años podía hacer uso de esta red social con solo agregar sus datos personales y contacto: nombre, apellido, correo electrónico, fecha de nacimiento y género; sin embargo, en este entonces aún no estaba en su auge, es decir, era conocido pero no como actualmente. Fue hasta el 2011, cuando el sitio despuntó como el mayor servidor de fotos en el mundo, ya que todos querían compartir lo que hacían en su diario vivir: las visitas que hacían, lugares que visitaban, los paseos con amigos, ya que para finales de ese mismo año, el número de usuarios de Facebook superaba los 350 millones y hoy en día es más del doble de personas que usan esta red social superando los 2 billones (Gonçalves, 2020).

\section{Red social}

De acuerdo con Raffino (2020) las redes sociales son sitios de Internet formados por comunidades de individuos con intereses o actividades en común (como amistad, parentesco, trabajo) y que permiten entre sus usuarios el intercambio de información. Los individuos no necesariamente se tienen que conocer previo a tomar contacto a través de una red social, sino que pueden hacerlo a través de ella, y ese es uno de los mayores beneficios de las comunidades virtuales.

El origen de las redes sociales es bastante reciente, se puede decir que surgen en 1995 con la creación de classmates.com, a manos del estadounidense Randy Conrads. Esta red social buscaba reunir excompañeros de colegio o universidades.

Luego, al ver que el proyecto era exitoso, comenzaron a aparecer nuevas redes que pretendían reunir amigos, y para el año 2003, ya se habían hecho populares sitios, como: LinkedIn y MySpace, con objetivos más específicos (Raffino, 2020). En la actualidad, estamos rodeados por plataformas digitales, son parte de la vida cotidiana, donde facilitan la comunicación y ayudan a satisfacer deseos y necesidades humanas, las redes sociales representan una enorme oportunidad de negocio e ingresos, por eso entre más atrayente sea la plataforma y el contenido, mejor será la respuesta de los usuarios. A continuación, se presentan las redes sociales más utilizadas en México. 


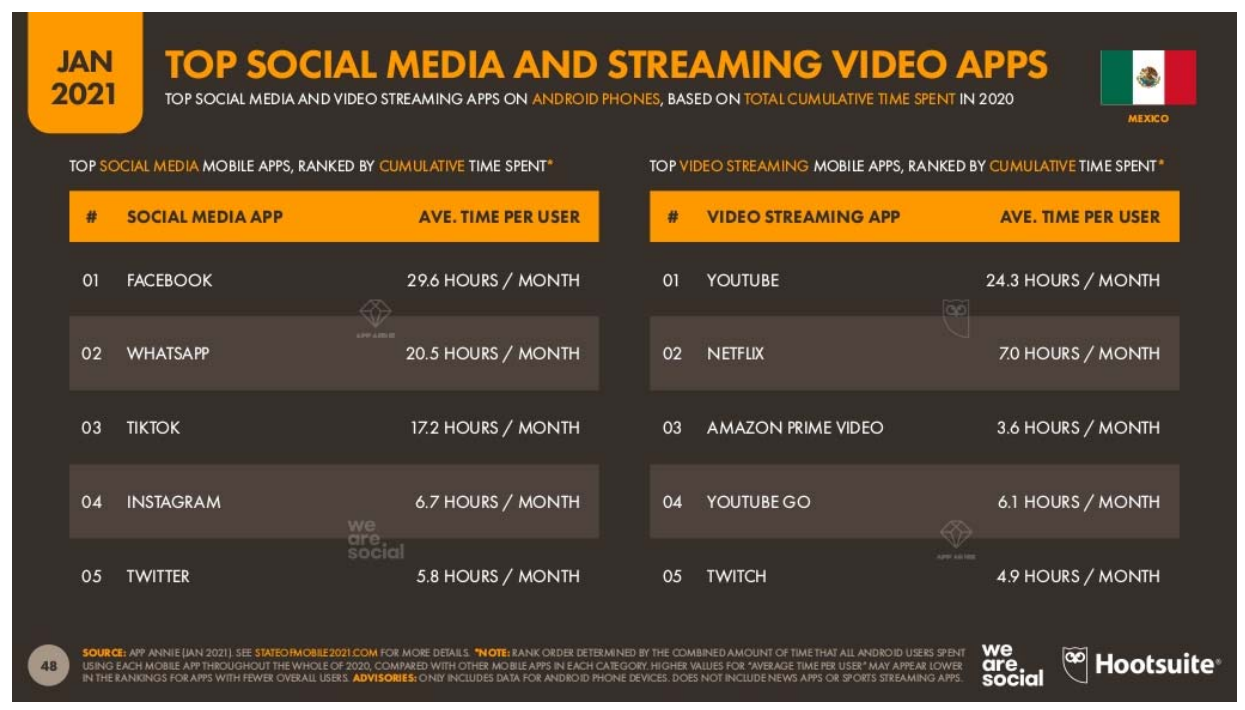

Figura 2. Top de aplicaciones de redes sociales y transmisión de video en México. Fuente: Datos tomados de Kemp, (2021).

De acuerdo a un reporte elaborado por el sitio Web Hootsuite, se puede apreciar en la Figura 2, que Facebook domina el mercado de las redes sociales en México, donde los usuarios utilizan un promedio acumulado de 29.6 horas al mes dicha aplicación, seguido por WhatsApp y en tercer lugar la plataforma TikTok.

\section{Publicidad digital}

De acuerdo con Galeano (2019), el marketing digital se puede definir como la aplicación de estrategias de comercialización llevadas a cabo en los medios digitales. También se define como el marketing interactivo, enfocado, medible, mediante el uso de tecnologías digitales con el propósito de alcanzar y crear prospecto de cliente en consumidores. El principal objetivo es, promover marcas, incrementar ventas, crear preferencia de un producto o servicio mediante el uso de diferentes técnicas de Marketing Digital, que coadyuven al incremento de adquisición.

Esta misma autora menciona, que en la actualidad prevalece el Marketing Digital, porque cuando se realiza una publicación a través del también conocido Marketing de Contenidos, se logra captar la atención del cliente potencial y se puede concretar la venta de manera indirecta hasta la entrega del producto. En este sentido, Galeano (2019), afirma que la tendencia del Marketing Digital, es el contenido audiovisual a través del consumo de videos en línea (esto puede variar dependiendo el usuario); sin embargo, esto quedará como una hipótesis hasta obtener los resultados de este estudio. 
LA MERCADOTECNIA EN LA RED SOCIAL "FACEBOOK".

Para entender mejor sobre este concepto, se tiene que hacer una pequeña retrospectiva a los 60's cuando Philip Kotler definió el Marketing Mix (las 4 P's) y como éste ha evolucionado en la actualidad. En este sentido, hay que recordar que las 4 P's son: Precio, Producto, Plaza y Promoción; sin embargo, en la actualidad esto ha cambiado, para lo cual Nager (2020) menciona que la primera evolución que tuvo el Marketing Mix fue que se le agregaron tres más, siendo ahora 7 P's, agregando Procesos, Personas (clientes) y Physical Evidence. Ahora bien, para la década de los 90's, Lauterborn las sustituyó por las 4C, porque dotó al marketing mix más Customer-driven (orientación al cliente); para ello, las modificaciones fueron las siguientes:

- Producto: Se convirtió en cliente, porque se determinó que las estrategias de marketing debían centrarse en el cliente y no en el producto.

- Precio: Ahora pasó a ser costo, puesto que el cliente debe tener las mejores facilidades para adquirir un producto o servicio.

- Plaza: Paso a ser conveniencia, esto porque como el cliente debe contar con las facilidades de adquirir un producto, también se le cede el derecho de decidir el canal y momento que desee para recibir su producto.

- Promoción: Evolucionó a comunicación, porque a través de ella se podrá escuchar al cliente y las necesidades que éste tiene para un producto específico.

La publicidad, es una forma de hacer comunicación, que pretende captar la atención del consumidor mediante mensajes creativos, para potencializar los beneficios de los productos que se producen, destacar sus bondades, generar atracción y convencimiento, ofreciendo al consumidor ciertos valores que pueden ser costo, beneficio o exclusividad (Cano, 2019, pp. 5-6).

\section{Principales ventajas del Marketing Digital o Publicidad Digital}

De acuerdo con Nager (2020), las principales ventajas del Marketing Digital son:

- Costos accesibles: los precios que se manejan en las ventas online pueden ser más barato que los que se publicitan en medios de comunicación como TV, Radio, Periódico.

- Mayor capacidad de control, optimización de ventas debido a la consulta que los usuarios realicen en tiempo real (interacción).

- Flexibilidad y dinamismo: de acuerdo con los resultados obtenidos en las ventas a través de la publicidad se pueden realizar cambios que ayuden a generar clientes. 
LA MERCADOTECNIA EN LA RED SOCIAL "FACEBOOK".

- Segmentación específica, personalizada y precisa: a través de una segmentación en el marketing online la empresa puede llegar a un determinado grupo de personas tomando en cuenta los datos sociodemográficos, psicológicos, socioeconómicos, edad, género, etc., lo cual disminuye gastos innecesarios y obtención de mejores ganancias.

\section{Cliente potencial}

Nuñez (2000), menciona que:

Es parte del mercado potencial. Puede verse utilizado en el sentido mercadotécnico, como objeto de la estrategia de mercadeo, aunque es más común que se use simplemente al término del cliente quedando tácito el sentido de potencialidad. En esta esfera no se alude comúnmente al cliente real o al potencial, al interno o al externo. Las estrategias se trazan para todos los potenciales. Algunos clientes, cuando han utilizado, comprado o consumido de los productos o servicios y lo hacen frecuentemente, se les llama clientes fijos. En resumen, el término cliente potencial es poco usado y en su lugar sin especificaciones se emplea la denotación cliente o mercado. Algunas deficiencias de traducción, fundamentalmente del inglés, generan confusión, ya que en ese idioma se emplean varios vocablos para el cliente (p. 114).

Como representación de las personas que perciben los esfuerzos de marketing en Facebook, se puede observar las diferentes edades en la siguiente Tabla.

\section{Tabla 1}

Edad de receptores de la publicidad en Facebook

\begin{tabular}{cc}
\hline Edad & Porcentaje \\
\hline $06-11$ & $1 \%$ \\
$12-17$ & $22 \%$ \\
$18-24$ & $21 \%$ \\
$25-34$ & $19 \%$ \\
$35-44$ & $12 \%$ \\
$45-54$ & 10 \\
+55 & $5 \%$ \\
\hline
\end{tabular}

Fuente: Elaboración propia.

Para poder tener una buena estrategia de marketing es necesario tener muy claro hacía que sector de la población está dirigida la publicidad. Conocer los perfiles, gustos y preferencias, 
LA MERCADOTECNIA EN LA RED SOCIAL "FACEBOOK".

edades, géneros, son solo una parte de la información, muchas veces también resulta pertinente conocer los horarios en que más personas acceden a la plataforma y el comportamiento que tienen los usuarios.

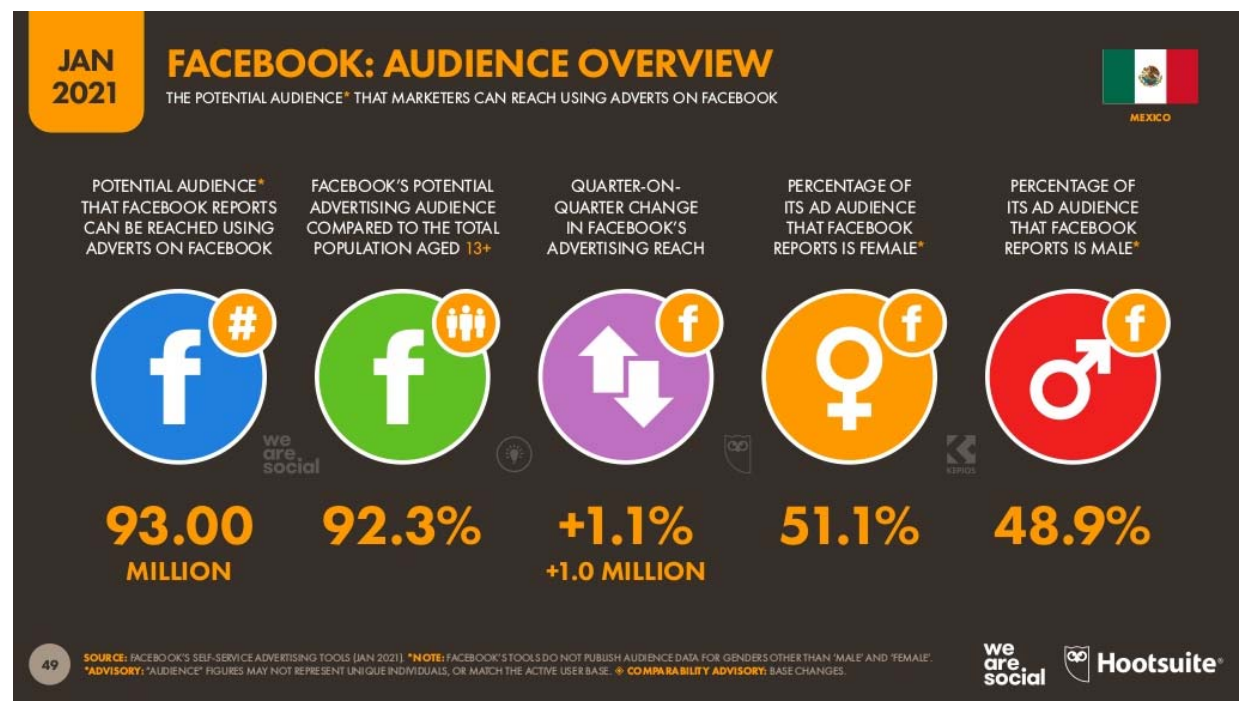

Figura 3. Facebook: descripción general de la audiencia.

Fuente: Datos tomados de Kemp, (2021).

En la Figura 3, se aprecian algunos datos muy relevantes como lo son: la audiencia potencial a la que se puede llegar a través de anuncios en Facebook en México es de 93 millones de personas, aproximadamente el $51.1 \%$ de la población que utiliza Facebook es público femenino y el $48.9 \%$ masculino.

De igual forma el estudio realizado expone que actualmente las personas han modificado los medios en que consumen contenido de Facebook, ya que aproximadamente el 98.9\% de los usuarios lo hacen mediante dispositivos móviles como Smartphones o Tablets y tan solo el 1.1\% lo hace mediante un equipo de cómputo (Kemp, 2021).

\section{E-Commerce}

De acuerdo con Carvazos y Reyes (2008), debido al uso de tecnologías cada vez más poderosas, junto con herramientas de mercadotecnia adaptadas al Internet, se ha incrementado la cantidad de transacciones comerciales a través de este medio, lo cual ha dado lugar al concepto de comercio electrónico e-Commerce.

Cavazos y Reyes (2008), mencionan que la mezcla de mercadotecnia en Internet debe integrarse por los siguientes puntos: 


\section{LA MERCADOTECNIA EN LA RED SOCIAL "FACEBOOK".}

1. Un producto personalizado.

2. Un precio flexible orientado hacia el valor para el consumidor.

3. Una distribución que elimine a los intermediarios.

4. Una promoción interactiva.

5. Procesos basados en el uso estratégico de TI (Tecnologías de Información).

6. Relaciones duraderas entre la empresa y el cliente.

Por su parte, Cruz (2009) un ejemplo exitoso del uso de las redes sociales por parte de las empresas es lo que está ocurriendo con las aerolíneas mexicanas VivaAerobús y Volaris, que se han dado cuenta de que pueden utilizar en forma estratégica las redes sociales, como: Facebook, Twitter, YouTube y Flickr. La empresa VivaAerobus, comenzó a utilizar las redes sociales en agosto del 2009, y con ello, ha logrado que un $20 \%$ de sus usuarios se mantenga en contacto con la empresa a través de este medio. En el caso de Volaris, incursionó en esta estrategia a principios del 2009 y es la que más presencia tiene en la red Twitter. Según José Calderoni, gerente de mercadotecnia de Volaris: "nos damos cuenta en un minuto qué problemas hay o qué interés hay en la página de Internet".

Para Ríos (2014), el comercio electrónico, puede clasificarse en:

\section{A. Las partes que interactúan:}

- $\quad$ B2B (Business to Business): Es aquel que se lleva a cabo entre dos empresas.

- B2C (Business to Consumer): Es el comercio de productos que se establece entre una empresa y un consumidor.

- C2C (Consumer to Consumer): Implica el comercio entre consumidores mediante una empresa que sólo funciona como soporte del intercambio.

B. La naturaleza de la cosa que se transfiere:

- Directa: La operación comercial se agota mediante el acto inicial de transferencia de la cosa intangible. La transferencia se caracteriza como perfecta.

- Indirecta: Implica la transferencia de una cosa tangible y requiere de una segunda etapa que es la entrega al comprador. Es un sistema que podríamos considerar como "imperfecto", pues la no inmediatez de la transacción puede producir inconvenientes jurídicos. 
LA MERCADOTECNIA EN LA RED SOCIAL "FACEBOOK".

\section{MATERIAL Y MÉTODO}

El estudio se realizará con el enfoque metodológico cuantitativo, este tipo de investigación utiliza la recolección de datos para probar o rechazar una hipótesis con base en la medición numérica lo que permite su representación de forma estadística y gráfica (Hernández, Fernández y Baptista, 2014), se plantea de esa manera una investigación de sondeo Flash Online en dicha red social por la naturaleza de la misma y de acuerdo con el objeto de estudio, para su comprensión y aplicación se deben tener resultados precisos a la hora de investigar de acuerdo con Arroba (2000).

Un sondeo flash es una encuesta relámpago; es decir, una encuesta de diez preguntas, que debe ser aplicada en un día, como máximo, esto no quiere decir que tenga que ser aplicada a un reducido número de personas, al contrario, respeta los estándares de un cuestionario de investigación, metodológica y estadístico, (Arroba, 2000).

\section{Muestra}

Se ha elegido para la investigación cualitativa una muestra aleatoria de 50 personas, usuarios de la misma red social. Las preguntas que se aplicaron son de opción múltiple, en las que los encuestados respondieron de acuerdo a su experiencia utilizando la red social de interés.

\section{Tabla 2}

Preguntas de encuesta flash

\section{Preguntas}

1. ¿Se ha fijado usted en algún anuncio publicitario en esta red social?

2. ¿En cuál red social ha visto más publicidad?

3. ¿Qué le ha llamado la atención del anuncio publicitario?

4. ¿Ha hecho clic en algún anuncio publicitario publicado en la red social?

5. ¿Lo han motivado a la compra los anuncios publicitarios?

6. ¿A qué cantidad de publicidad en las redes sociales siente que es expuesto al día?

7. ¿Usaría la red social Facebook para hacer publicidad sobre algún producto o servicio?

Fuente: Elaboración propia. 
LA MERCADOTECNIA EN LA RED SOCIAL "FACEBOOK".

RESULTADOS

Los resultados obtenidos en el sondeo Flash Online, relacionado con los diversos usuarios de la red social Facebook, aptos para percibir las diversas publicidades son los siguientes, a continuacion se muestran las Figuras que dio como resultado el sondeo flash de cada cuestionamiento.

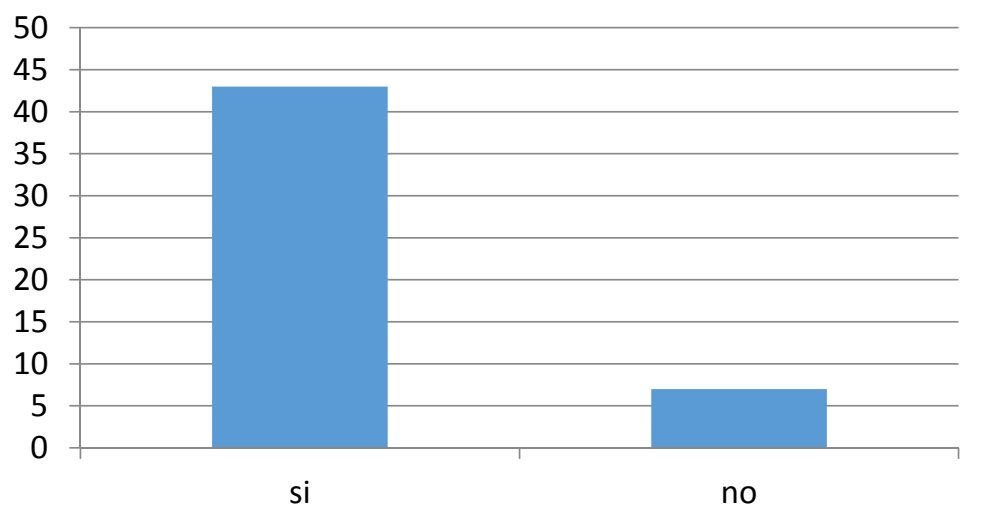

Figura 4. ¿Se ha fijado usted en algún anuncio publicitario en esta red social? Fuente: Elaboración propia.

De acuerdo con la encuesta realizada a una muestra de 50 personas para realizar un estudio rápido del impacto de la publicidad en la red social, la población analizada si respondió a favor de la efectividad de la publicidad en Facebook, y los gastos que hacen en compras y promociones que encuentran en dicha red.

En este sentido, en la Figura 4, 43 personas encuestadas (lo que representa el 86\% de la muestra) afirman que si se fijan en un anuncio publicitario en Facebook y solo el $14 \%$ ( 7 personas) hacen caso omiso de los anuncios que se hagan en esta red social; es decir, que el impacto de una publicidad es demasiado alto.

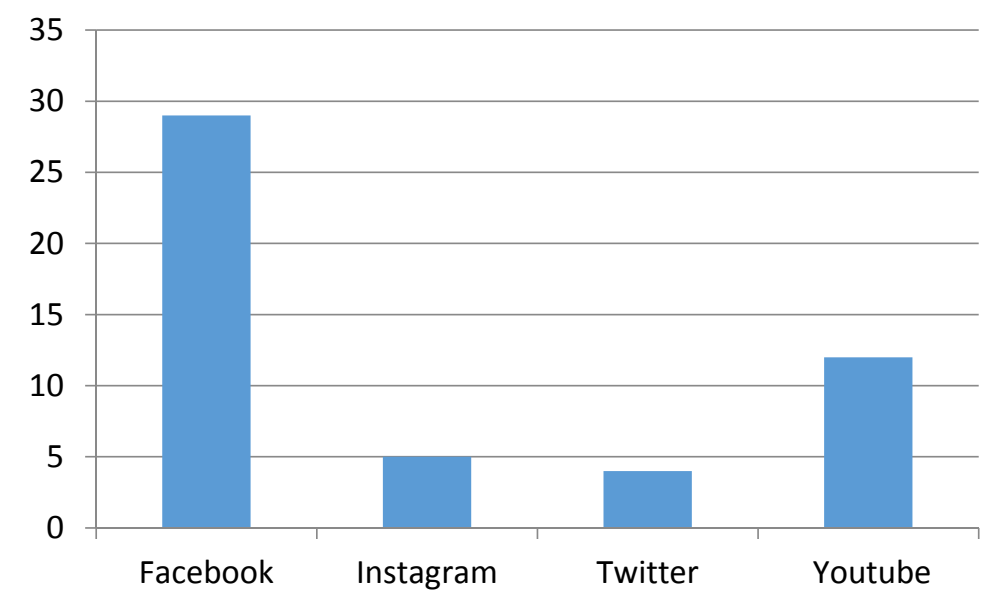

Figura 5. ¿En qué red social has visto más publicidad?

Fuente: Elaboración propia.

FUENTES-FRÍAS F. A., GARCÍA-RODRÍGUEZ J. F. HITOS DE CIENCIAS ECONÓMICO ADMINISTRATIVAS 
LA MERCADOTECNIA EN LA RED SOCIAL "FACEBOOK".

Por otra parte, en la Figura 5, los usuarios tuvieron división de opiniones, dando como resultado lo siguiente: el 10\% (5 personas) respondió que ha visto anuncios publicitarios en Instagram; el 8\% (4 personas) mencionan que han visto publicidad en Twitter, siendo esta red social la menor impacto en cuanto a anuncios publicitarios, ya que es la que menos usuarios utilizan; el 24\% (12 personas) argumentan que mientras visualizan un video o película en YouTube han tenido que ver un anuncio publicitario si no cuentan con una cuenta premium; y, por último, el 58\% (29 personas) afirma que ha visto una publicidad mientras navega en Facebook, lo cual confirma que esta red social es la de mayor impacto para realizar anuncios de productos y/o servicios.

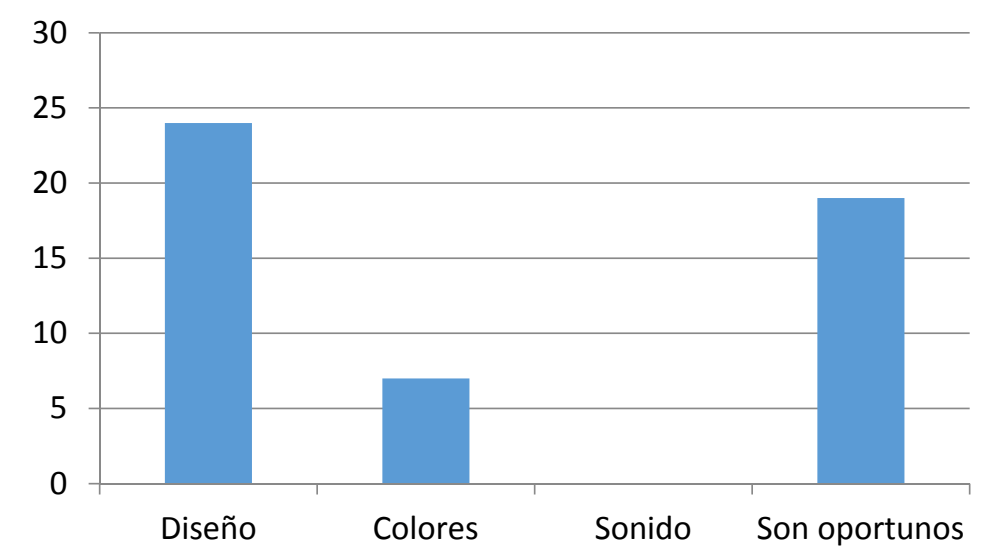

Figura 6. ¿Qué le ha llamado la atención del anuncio publicitario? Fuente: Elaboración propia.

En la Figura 6, el 14\% (7 personas) se fijan en los colores que se muestran en la publicidad, el $38 \%$ (19 personas) toman en cuenta lo oportuno que el anuncio puede ser en el momento que lo visualicen; es decir, si están necesitando un artículo del hogar y en ese instante aparece el de una tienda departamental, es muy probable que se inclinen por adquirir el producto; como siguiente resultado, el 48\% (24 personas) afirman que lo más importante de un anuncio publicitario es el diseño que éste tenga, lo atractivo que puede llegar a ser, y, por último, el sonido que contenga la publicidad, siendo el $0 \%$ de esta muestra que toman en cuenta dicha característica. En otras palabras, se deduce que en lo que más se fijan los usuarios en Facebook de un anuncio publicitario, es el diseño; sin embargo, en conjunto todo puede ser de ayuda para que el cliente potencial adquiera el producto. 
LA MERCADOTECNIA EN LA RED SOCIAL "FACEBOOK".

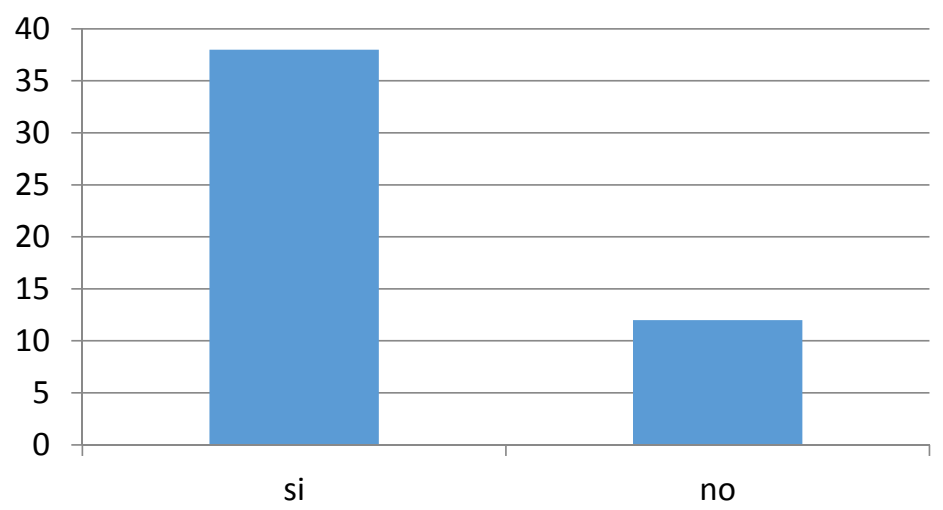

Figura 7. ¿Ha hecho clic en algún anuncio publicitario publicado en la red social? Fuente: Elaboración propia.

En la Figura 7, el 22\% (11 personas) respondió que no se han dado el tiempo de dar clic en un anuncio publicitario en Facebook, mientras que el 78\% (39 personas) si han sentido el interés de dar clic en la publicidad, esto, en consecuencia, de la pregunta anterior; es decir, si no muestra un diseño atractivo, el color no tiene nada que ver con lo que anuncian o no les interesa adquirir el producto mostrado, simplemente no se inclinan por saber más de él.

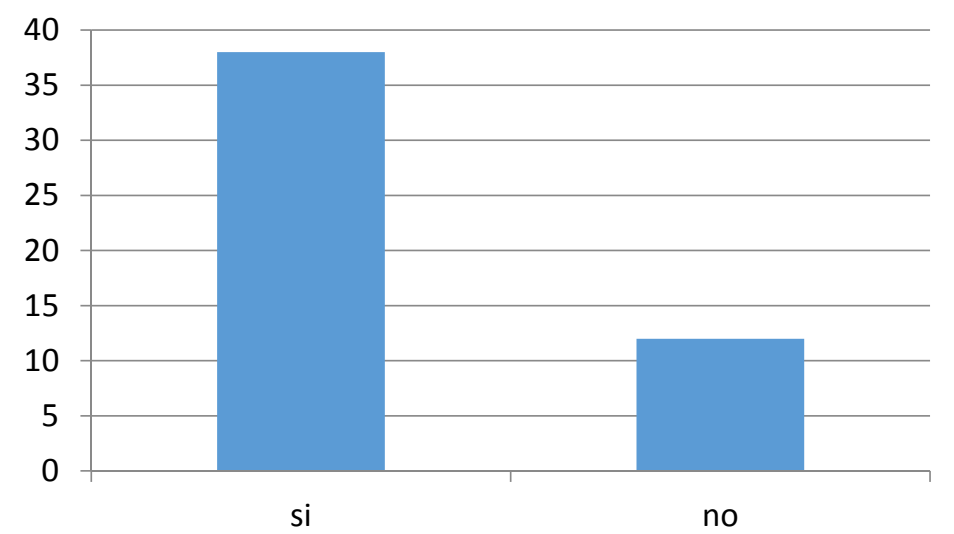

Figura 8. ¿Lo han motivado a la compra los anuncios publicitarios?

Fuente: Elaboración propia.

En la Figura 8, se les cuestionó si han sido motivados por la publicidad para adquirir el producto, dando como resultado que el 24\% (12 personas) no han sentido motivación alguna por lo que les venden en esta red social mediante un anuncio, mientras que el $76 \%$ (38 personas) si se han sentido motivados para adquirir el producto o servicio que en ese momento visualizaron en Facebook. En otras palabras, se confirma que existe un grado de impacto en la motivación de un individuo para la adquisición de compra a través de esta red social. 
LA MERCADOTECNIA EN LA RED SOCIAL "FACEBOOK".

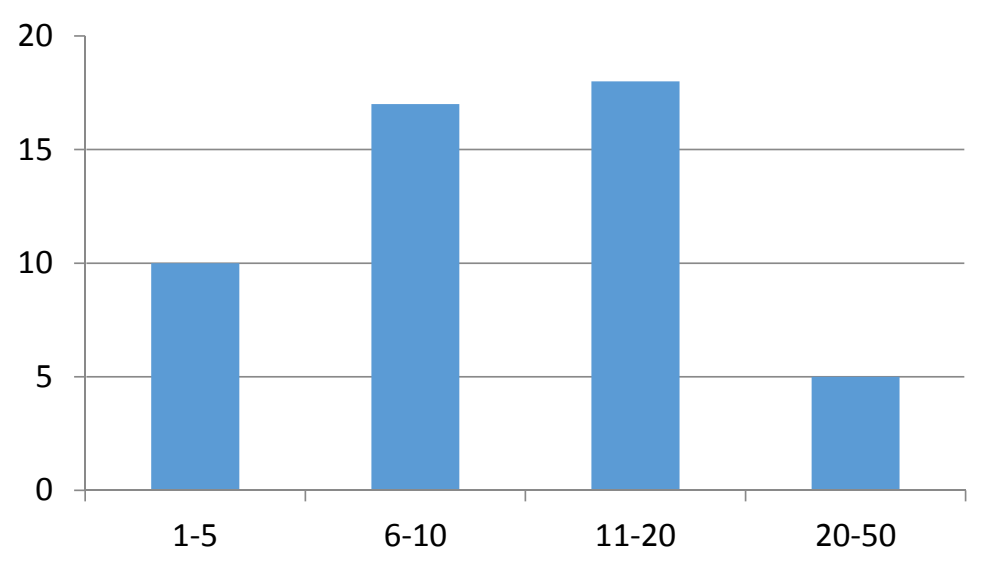

Figura 9. ¿A qué cantidad de publicidad en las redes sociales, siente que es expuesto al día?

Fuente: Elaboración propia.

En la figura 9, el 10\% (5 personas) afirma que han visto más de 20 anuncios mientras navegaba en esta red social; el 20\% (10 personas) respondió que ha visto de 1-5 anuncios estando en Facebook, el 34\% (17 personas) argumenta que ha visto de 6-10 anuncios, y, por último, el $36 \%$ (18) menciona que ha visto de 11-20 anuncios en esta red social, lo que confirma que una persona en promedio debe ver más de 10 anuncios publicitarios en Facebook para poder adquirir un producto o servicio; o bien, visualizar la misma publicidad varias veces para motivarse a comprar.

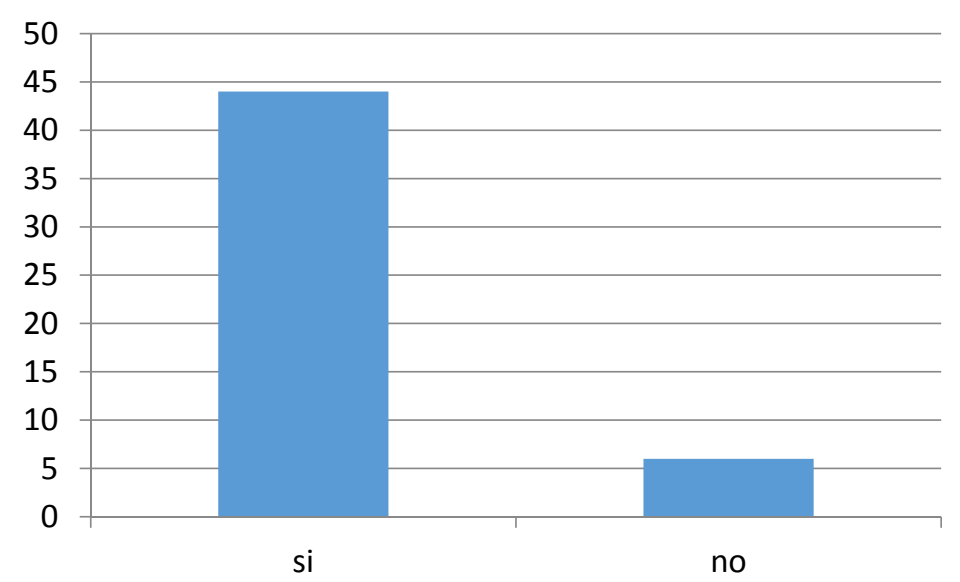

Figura 10. ¿Usaría la red social Facebook para hacer publicidad sobre algún producto o servicio?

Fuente: Elaboración propia.

Por último, en la Figura 10, el 12\% (6 personas) no la usarían y solo comprarían lo que les venden (si se les hace atractivo o necesario), y el 88\% (44 personas) afirman que usarían a Facebook como medio publicitario para la venta de sus productos o servicios. En otras 
LA MERCADOTECNIA EN LA RED SOCIAL "FACEBOOK".

palabras, aunque hay diferentes perspectivas de uso de esta red social, hoy en día la mayoría hace uso de ella para realizar una venta por el alcance que tiene y el impacto que genera en los usuarios.

\section{CONCLUSIONES}

Después de haber realizado el análisis cualitativo en cuando a la publicidad en Facebook y el impacto que genera en los usuarios de ésta, se puede afirmar satisfactoriamente que hoy en día si se quiere realizar una compra/venta de productos y/o servicios, esta red social es la más apropiada por el alcance que tiene a nivel mundial porque hay que recordar que al instante que realizas la publicación puedes llegar al otro lado del mundo con solo un clic, lo que la hace totalmente viable para utilizarla.

Ahora bien, de acuerdo con los resultados hay que tomar en cuenta que no solo es realizar un anuncio publicitario, sino que hay que tomar en cuenta algunos puntos para que la publicidad sea atractiva al cliente potencial; es decir, colores llamativos, lo oportuno que puede ser, el diseño que se utilizó para publicitar, a las personas que se quiere llegar, porque si esto se pasa por alto, será una publicación más sin respuesta positiva de compra. Cabe señalar que, lo que menos toman en cuenta los usuarios es el sonido de un anuncio (de acuerdo con los resultados de la encuesta), lo que quiere decir, que no es tan viable realizar videos para anunciar un producto, por el contrario, que sean posters, imágenes, fotografías reales del producto, que den más credibilidad a lo que se ofrecerá en la página o perfil de Facebook.

Con lo anterior, se determina que la publicidad en esta red social, es una oportunidad de inversión para cualquier usuario, por lo que se considera totalmente factible.

\section{REFERENCIAS BIBLIOGRÁFICAS}

Arroba, J. (2000). ¿Cuándo y cómo se hace un sondeo flash? Chasqui(70), 42-46. doi:https://doi.org/10.16921/chasqui.v0i70.1355

Cano, M. (2019). La evolución de la publicidad tradicional a la publicidad digital: el caso de la publicidad automotriz en México 2007-2017. (Tesis para obtener el grado de Licenciado en ciencias de la comunicación). UNAM, México. 
LA MERCADOTECNIA EN LA RED SOCIAL "FACEBOOK".

Carvazos, J. y Reyes, S. (2008). Comercio electrónico: un enfoque de modelos de negocio. México: Grupo Editorial Patria.

Cruz, L. (12 de Noviembre de 2009). Resulta eficaz contacto virtual con clientes. Periódico AM, p. 8.

Galeano, S. (11 de Junio de 2019). Definición de marketing digital, su historia, objetivos y sus grandes tendencias. M4rketing ecommerce MX. Recuperado de https://marketing4ecommerce.mx/marketing-digital-definicion-historia/

Garibay, J. (2017). ¿Qué le depara al marketing digital en el mundo? Revista 2.0. Recuperado de https://www.merca20.com/que-le-depara-al-marketing-digital-en-el-mundo/

Gonçalves, W. (13 de Marzo de 2020). Facebook: ¡Todo sobre la red social más usada en el mundo! RockContecnt. Recuperado de https://rockcontent.com/es/blog/facebook/

González, F. (2018). Efectividad de la publicidad en Facebook: ¿Qué industrias lo hacen mejor en Latinoamerica? Merca 2.0. Recuperado de https://www.merca20.com/efectividad-dela-publicidad-en-facebook-que-industrias-lo-hacen-mejor-en-latinoamerica/

Hernández, R., Fernández, C. y Baptista, M. (2014). Metodología de la investigación. México, D.F.: McGraw-Hill Education.

Kemp, S. (2021). Digital 2021: México. Recuperado de https://datareportal.com/reports/digital2021-mexico

Nager, E. (30 de Junio de 2020). ¿Qué es el Marketing Digital o Marketing Online? INBOUNCYCLE. Recuperado de https://www.inboundcycle.com/blog-de-inboundmarketing/que-es-el-marketing-digital-o-marketing-online

Newberry, C. (19 de Febrero de 2020). 33 estadísticas de Facebook que todo mercadólogo debe conocer en 2020. Hootsuite. Recuperado de https://blog.hootsuite.com/es/estadisticas-de-facebook/

Nuñez, I. (2000). Usos y definiciones de los términos relativos a los usuarios o clientes. Revista Interamericana de Medellín Colombia, 23(1), 107-121. Recuperado de https://revistas.udea.edu.co/index.php/RIB/article/view/7926/7435

Raffino, M. E. (23 de Septiembre de 2020). Concepto de Redes Sociales. Concepto.de. Recuperado de https://concepto.de/redes-sociales/

Ríos, A. (2014). Análisis y perspectivas del comercio electrónico en México. Enlace Revista Venezolana de Información, Tecnología y Conocimiento. 11 (1), 103. 